\title{
Fibrillazione atriale e malattia renale cronica
}

\author{
Luca Di Lullo ${ }^{1}$, Fulvio Floccari ${ }^{2}$, Antonio De Pascalis ${ }^{3}$, Vincenzo Barbera ${ }^{1}$, \\ Giovanni Barbera ${ }^{1}$, Moreno Malaguti ${ }^{2}$, Alberto Santoboni ${ }^{1}$
}

${ }^{1}$ U.O.C. Nefrologia e Dialisi, Ospedale L. Parodi-Delfino, Colleferro (RM)

${ }^{2}$ U.O.C. Nefrologia e Dialisi, Ospedale S. Paolo, Civitavecchia (RM)

${ }^{3}$ U.O.C. Nefrologia, Dialisi e Trapianto, Ospedale V. Fazzi, Lecce

\begin{abstract}
Atrial Fibrillation AND CHRONIC KIDNEY DISEASE
Abstract. Atrial fibrillation represent the most common type of arrhythmia encountered in clinical practice, especially in chronic ischemic heart disease patients and in chronic kidney disease (CKD) patients, and is often correlated to increased age and related comorbidities.

CKD patients often present several comorbidities, such as hypertension, diabetes, and vascular disease, and they often present arrhythmias that are usually linked to their related cardiomyopathy.

During the last 2 decades, an increase in the prevalence of atrial fibrillation among CKD patients, especially those on hemodialysis treatment, was reported.
\end{abstract}

Key words: Chronic kidney disease, Dialysis, Dilated cardiomyopathy, Atrial fibrillation

Conflict of interest: None.

Financial support: None.

Accettato: 3 Febbraio 2014

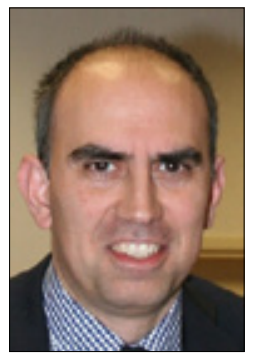

Luca Di Lullo

\section{Introduzione}

La fibrillazione atriale rappresenta la più comune aritmia riscontrabile nella nostra pratica clinica quotidiana.

Questo è valido soprattutto per i pazienti cardiopatici e, in particolare, per quelli affetti da diverse forme di cardiomiopatia dilatativa (1) e da cardiopatia ischemica cronica (1).

L'incidenza e la prevalenza della fibrillazione atriale si presentano in costante aumento anche nella popolazione affetta

da malattia renale cronica, probabilmente anche in relazione con l'aumentata incidenza, in questi pazienti, di diverse comorbidità come l'ipertensione arteriosa, il diabete mellito e la vasculopatia polidistrettuale. Studi di epidemiologia clinica hanno documentato anche un aumento dei casi di fibrillazione atriale strettamente correlati all'aumento dell'età media, sia della popolazione generale sia dei pazienti con malattia renale cronica (2).

Tra questi ultimi, sono da menzionare, in particolare, i pazienti sottoposti al trattamento emodialitico, nei quali le variazioni delle concentrazioni elettrolitiche plasmatiche giocano un ruolo di primaria importanza (3-7).

La malattia renale cronica (Chronic Kidney Disease, CKD) e le patologie cardiovascolari condividono diversi fattori di rischio, come accennato in precedenza: il diabete mellito, l'ipertensione arteriosa e uno stato di infiammazione cronica rappresentano solo alcune delle comorbidità in comune tra le due entità nosologiche.

Più di recente, l'evidenza di una stretta correlazione tra malattia renale cronica e patologia cardiovascolare ha portato a individuare quella che ormai è comunemente definita sindrome cardio-renale $(8,9)$.

Questo stretto legame ha, inoltre, assunto particolare valore alla luce anche di importanti implicazioni di tipo prognostico, soprattutto nella popolazione di pazienti over $75(8,9)$.

L'incidenza della fibrillazione atriale è particolarmente aumentata, nelle ultime due decadi, nei pazienti sottoposti al trattamento emodialitico (10).

\section{Prevalenza e incidenza}

Come anticipato nell'introduzione, la fibrillazione atriale rappresenta l'aritmia di più frequente riscontro nella pratica clinica quotidiana; studi di epidemiologia clinica, infatti, ne stimano una prevalenza mondiale variabile dallo $0.5 \%$ all' $1 \%$, con le nuove diagnosi effettuate per la maggior parte nei soggetti che hanno superato i 75 anni di età.

Prima dei 50 anni, le statistiche evidenziano una prevalenza pari allo $0.5 \%$, mentre nel corso dell'ottava decade di vita si 
arriva a livelli di prevalenza pari all' $8 \%$ (4).

Se osserviamo più da vicino la coorte di pazienti affetti da malattia renale cronica terminale e sottoposti al trattamento emodialitico, sono stati riportati dati di prevalenza di fibrillazione atriale variabili dal $10 \%$ al $27 \%$ (10); stime abbastanza simili sono state ottenute anche in pazienti affetti da malattia renale cronica, ma ancora in fase pre-terminale (10-12).

Se consideriamo, come riferimento, la popolazione generale, la prevalenza della fibrillazione atriale nei pazienti affetti da malattia renale cronica agli stadi 3-5 e in trattamento sostitutivo della funzione renale è superiore di $10-15$ volte (10).

Fattori di rischio indipendenti per la presenza di fibrillazione atriale in pazienti sottoposti al trattamento dialitico sono sicuramente rappresentati dall'età biologica, dall'età dialitica, dalla presenza di atriomegalia in pazienti già affetti da cardiomiopatia dilatativa, dal sesso maschile, dalla presenza di una malattia coronarica, dal coinvolgimento delle sezioni destre (e, più in particolare, dalla presenza di un quadro di dilatazione atriale destra), da un basso indice di Karnofsky, da un alto valore di BMI, dalla presenza di diabete mellito e/o di scompenso cardiaco cronico e da una storia di pregressi eventi cerebrovascolari (7).

Nei pazienti affetti da malattia renale cronica agli stadi 3-4, un'ulteriore riduzione della frazione di filtrazione glomerulare rappresenta un fattore predittivo indipendente di aumento di prevalenza della fibrillazione atriale, come riportato in studi di comunità effettuati su pazienti sani e pazienti cardiopatici in fase di compenso emodinamico $(10,13-16)$.

Studi clinici di tipo longitudinale effettuati in pazienti sottoposti a trattamento emodialitico e condotti in un arco di tempo variabile tra 1 anno e 7 anni hanno riportato un'incidenza della fibrillazione atriale in un range variabile da 1 a 5.9 casi/100 pazienti/anno $(10,17-21)$.

Se si paragonano questi dati con quelli provenienti da studi epidemiologici, l'incidenza di nuovi casi di fibrillazione atriale si presenta da 6 a 12 volte maggiore nei pazienti emodializzati che nella popolazione generale (10). Diversi fattori di rischio sono stati chiamati in causa per l'elevata incidenza della fibrillazione atriale in questa particolare popolazione di pazienti: età avanzata, ipertrofia ventricolare sinistra, mancata assunzione di ACE-inibitori e/o AT-II antagonisti, pressioni di incuneamento capillare più elevate, storia di eventi acuti cerebrovascolari, ritardi di conduzione atrio-ventricolare, calcificazioni valvolari, ridotta frazione di eiezione (indice, a sua volta, di ridotta compliance ventricolare sinistra) e presenza di anemia secondaria (10).

\section{Fisiopatologia}

La fibrillazione atriale è caratterizzata dalla presenza di una condizione, a livello elettrofisiologico, di caotica depolarizzazione atriale che comporta, a sua volta, una disorganizzazione nel processo di contrazione atriale.

La fibrillazione atriale viene facilmente diagnosticata all'esame elettrocardiografico ma sono tuttora parzialmente sconosciute le dinamiche fisiopatologiche che sottintendono l'instaurarsi di tale aritmia.

L'associazione della fibrillazione atriale con diversi altri qua- dri clinici testimonia un'eziopatogenesi quanto mai eterogenea e alcuni probabili meccanismi fisiopatologici alla base del processo sono stati studiati in modelli animali (22).

Da diverso tempo è risaputo come una condizione di fibrillazione atriale possa essere scatenata dalla depolarizzazione spontanea del tessuto miocardico presente nelle vene polmonari (23).

È stato, inoltre, dimostrato come l'isolamento elettrico delle vene polmonari mediante ablazione a radiofrequenza (RFA) sia in grado di porre fine allo stimolo aritmico e di prevenire delle recidive di fibrillazione atriale (23).

Studi clinici successivi hanno evidenziato la presenza di punti precisi presenti sulla superficie dell'atrio destro in grado di facilitare la persistenza di uno stimolo di natura aritmogena a supporto del concetto secondo il quale vi sarebbero, a livello dell'atrio destro, delle regioni "trigger" in grado di stimolare l'insorgenza di un quadro aritmico (24).

È stato anche evidenziato come l'ablazione elettiva di queste zone "trigger" sia in grado di ridurre in maniera significativa le recidive della fibrillazione atriale (24).

Elevate concentrazioni sieriche di diversi marker pro-infiammatori (proteina C-reattiva o PCR, interleuchina-6 o IL-6, tumor necrosis factor- $\alpha$ o TNF- $\alpha$ ) sono state associate alla maggiore incidenza della fibrillazione atriale ed è stato postulato come dette concentrazioni siano direttamente correlate alla frequenza delle recidive della fibrillazione atriale dopo una cardioversione elettrica (24-26).

Alcuni trial clinici hanno, inoltre, documentato, l'efficacia della terapia steroidea nella prevenzione della fibrillazione atriale conseguente a interventi di cardiochirurgia (27).

In fisiologia è ben risaputo come, alla base della stabilità delle membrane cellulari dei miocardiociti e della corretta trasmissione dell'impulso elettrico, ci debba essere un corretto funzionamento dei canali ionici intracellulari, accompagnato dall'integrità dei complessi delle proteine strutturali transmembrana (denominate "connessine") e dall'omogeneità delle cariche elettriche della matrice extra-cellulare (28).

Studi sperimentali hanno evidenziato come citochine (TNF- $\alpha$ e IL-6, tra le altre) e radicali ossigenati (ROS) rilasciati a livello leucocitario, sotto l'influenza di citochine ad azione pro-infiammatoria e di altri ormoni, siano in grado di destabilizzare i canali ionici intracellulari e i complessi proteici trans-membrana, determinando alterazioni significative dei fenomeni di depolarizzazione/ripolarizzazione di membrana. Le citochine sopra citate, inoltre, sono in grado di promuovere fenomeni di fibrosi extracellulare e di determinare fenomeni di rimodellamento elettrico e strutturale del tessuto di conduzione atriale; il risultato finale è quello di provocare una maggiore suscettibilità all'insorgenza della fibrillazione atriale (27).

Nei pazienti affetti da malattia renale cronica, è stata evidenziata la presenza di aumentati livelli di ROS e di citochine ad azione pro-infiammatoria $(28,29)$; in modelli animali di malattia renale cronica e fibrillazione atriale, è stata evidenziata un'aumentata sintesi di ROS e di angiotensina II (AT-II) con il conseguente quadro di fibrosi interstiziale a livello atriale sinistro.

È stato anche dimostrato come l'infusione di agenti antiossidanti (Vitamina E) sia in grado, in modelli animali, di inibire 
la sintesi di ROS e di AT-II e, conseguentemente, di ridurre i fenomeni aritmici a livello del tessuto atriale (30).

Le precise dinamiche fisiopatologiche alla base dell'induzione e del mantenimento di un quadro di fibrillazione atriale nei pazienti affetti da malattia renale cronica non sono, tuttavia, ancora del tutto chiarite ma, sulla base delle evidenze sperimentali sin qui raccolte, sembra ragionevole pensare a un ruolo esercitato dal ROS e dalle citochine ad azione pro-infiammatoria nella patogenesi della fibrillazione atriale in corso di malattia renale $(30)$.

\section{Tromboembolismo}

Sia la fibrillazione atriale che la malattia renale cronica rappresentano condizioni cliniche caratterizzate da uno stato di ipercoagulabilità in grado di incrementare il rischio di accidenti cerebrovascolari acuti.

La presenza di una fibrillazione atriale si associa a un rischio 3-5 volte superiore che nella popolazione normale di eventi cerebrovascolari acuti, mentre la malattia renale cronica terminale, da sola, incide con un rischio pari a 4-10 volte quello della popolazione di controllo (31-33).

Studi clinici effettuati in pazienti sottoposti a un trattamento emodialitico con fibrillazione atriale hanno documentato un rischio di eventi cerebrovascolari acuti pari a 5-17 volte quello presente negli stessi pazienti ma non affetti da fibrillazione atriale $(34,35)$.

I punteggi $\mathrm{CHADS}_{2}$ (Congestive heart failure, Hypertension, Age $\geq 75$ years, Diabetes mellitus, previous Stroke/transient ischaemic attack (doubled risk weight)) e CHA $\mathrm{DS}_{2} \mathrm{VAS}_{\mathrm{c}}$ (Congestive heart failure, Hypertension, Age $\geq 75$ years, Diabetes mellitus, previous Stroke/transient ischaemic attack, Vascular disease, Age 65-74 years, Sex category; age $\geq 75$ years and previous stroke carry doubled risk weight) sono stati utilizzati per la quantificazione del rischio di eventi cerebrovascolari acuti associati alla fibrillazione atriale (in assenza di danni valvolari cardiaci) $(36,37)$.

E stato dimostrato che, aggiungendo agli score sopra citati un valore di creatinina clearance (valutata secondo la formula CKD-EPI) $<60 \mathrm{~mL} / \mathrm{min}$, si ha un ulteriore aumento del rischio di fibrillazione atriale nei pazienti nefropatici (38).

\section{Mortalità}

La presenza di fibrillazione atriale è in grado, nella popolazione generale, di determinare un aumento della mortalità di 1.51.9 volte rispetto ai soggetti non affetti (39); nei pazienti sottoposti al trattamento dialitico e affetti da fibrillazione atriale, sono stati evidenziati tassi di mortalità annuali pari al $25 \%$ $(10,40)$. Nei pazienti sottoposti al trattamento emodialitico, non affetti da fibrillazione atriale, la mortalità sembra essere da 1.9 a 3 volte inferiore rispetto alla stessa popolazione affetta da fibrillazione atriale cronica (10).

La presenza di fibrillazione atriale, cardiopatia ischemica cronica, diabete mellito e valvulopatie mitro-aortiche in pazienti sottoposti al trattamento dialitico è associata a un aumento della mortalità per cause cardiovascolari di almeno 2.5 volte; al contrario, età superiore ai 65 anni ed età dialitica rappresen- tano predittori indipendenti di mortalità per cause non cardiovascolari nella stessa popolazione di pazienti $(10,18)$.

Uno studio basato sull'analisi di 12 anni del registro danese dei pazienti affetti da fibrillazione atriale ha evidenziato i seguenti tassi di mortalità: $11.21 \%$ nei pazienti con funzione renale normale, $38.55 \%$ nei pazienti con malattia renale cronica agli stadi $2-4$ e $29.35 \%$ nei pazienti sottoposti a trattamenti sostitutivi della funzione renale (35).

\section{Trattamento}

Il trattamento farmacologico della fibrillazione atriale nei pazienti affetti da malattia renale cronica non differisce, sostanzialmente, da quello che si attua in pazienti con una funzione renale conservata.

Tutti i pazienti che presentano un quadro di fibrillazione atriale (specialmente se parossistica) associato a instabilità emodinamica o a una sindrome coronarica acuta dovrebbero essere immediatamente trattati con la cardioversione elettrica, indipendentemente dai parametri di funzionalità renale, anche se, da dati presenti in letteratura, risulta che l'incidenza di recidiva della fibrillazione atriale dopo cardioversione elettrica è superiore nei pazienti con malattia renale che nei pazienti che hanno una funzione renale preservata (41).

Va subito sottolineato come l'impiego di farmaci anti-aritmici sia associato a un più alto tasso, rispetto alla cardioversione elettrica, di eventi avversi $(42,43)$.

Nei pazienti sottoposti al trattamento emodialitico, l'incidenza di eventi avversi farmaco-correlati è maggiore, a causa dell'accumulo dovuto alla ridotta capacità, da parte dei pazienti dializzati, di eliminare i farmaci per via renale. Altri motivi che rendono difficoltoso l'impiego dei farmaci anti-aritmici nei pazienti sottoposti alla terapia dialitica sono costituiti dalle interazioni che avvengono tra i metaboliti degli stessi farmaci e dagli squilibri elettrolitici che avvengono normalmente durante il trattamento emodialitico. È stato persino segnalato l'arresto cardiaco in pazienti dializzati, moderatamente iperkaliemici, trattati con verapamil (44).

Per quanto riguarda l'impiego di digitale, questa può accumularsi rapidamente nei pazienti sottoposti al trattamento dialitico e può diventare estremamente tossica in presenza di oscillazioni significative della kaliemia nel corso della seduta emodialitica (45).

Nei pazienti emodializzati sottoposti alla terapia con digitale, l'allungamento dell'intervallo QTc, documentabile con un elettrocardiogramma di base, è stato correlato con l'aumentata incidenza di morte cardiaca improvvisa (46).

In caso di impiego di amiodarone, anche se esso non è associato a un aumento della mortalità nei pazienti emodializzati, questi ultimi vanno monitorati per prevenire un'eventuale alterazione dell'intervallo QTc alla luce del potenziale rischio di sviluppare una tachicardia ventricolare polimorfica, soprattutto nei pazienti con concomitante ipokaliemia (47).

L'ablazione trans-catetere di vie di conduzione anomala risulta essere sicuramente più efficace, rispetto alla terapia farmacologica, nel ripristinare il ritmo sinusale e nel prevenire le recidive di fibrillazione atriale $(48,49)$; tale procedura sembra, inoltre, molto più efficace anche nella prevenzione di eventi 
cerebrovascolari acuti rispetto al trattamento con farmaci antiaritmici (50), ma queste evidenze sembrano essere particolarmente valide per i pazienti con funzione renale conservata ovvero con malattia renale cronica pre-terminale. Nei pazienti sottoposti al trattamento dialitico, l'ablazione trans-catetere sembra, al contrario, essere associata a una maggiore incidenza di recidive (51).

\section{Terapia anticoagulante}

Come già discusso nel paragrafo dedicato alla fisiopatologia, la presenza di fibrillazione atriale, associata o meno a una condizione di malattia renale cronica, si accompagna a un elevato rischio di ictus e di tromboembolismo periferico.

La terapia anticoagulante cronica rappresenta, dunque, la pietra miliare del trattamento farmacologico nei pazienti affetti da fibrillazione atriale cronica, allo scopo di ridurre il rischio di tromboembolismo periferico e dei conseguenti eventi cerebrovascolari acuti.

La terapia a lungo termine con warfarin è in grado di ridurre del $60 \%$ il rischio di ictus nei pazienti affetti da fibrillazione atriale (52). Il profilo di sicurezza e di efficacia del warfarin, a proposito della prevenzione degli eventi cerebrovascolari acuti, non è stato ancora completamente valutato nei pazienti affetti da malattia renale cronica (32) e, a tutt'oggi, l'impiego del farmaco è oggetto di controversie nel caso di coesistenza di fibrillazione atriale e di deficit della funzione renale.

Studi clinici ormai datati avevano evidenziato come l'impiego di warfarin in pazienti sottoposti al trattamento dialitico fosse, in realtà, associato a un incremento del rischio di sviluppare ictus sia su base ischemica che su base emorragica (52). Studi più recenti, al contrario, supportano l'impiego del warfarin nei pazienti dializzati, evidenziando una significativa riduzione dell'incidenza di ictus, anche se accompagnata, rispetto alla popolazione con funzione renale conservata, da tassi più elevati di sanguinamento $(53,54)$.

Il rischio di sanguinamento associato all'impiego di warfarin risulta essere più elevato nei pazienti affetti da malattia renale cronica anche quando il valore di INR (International Normalized Ratio) viene mantenuto all'interno dell'intervallo terapeutico (55).

Gli inibitori diretti della trombina (dabigatran) e del fattore Xa (rivaroxaban, apixaban ed edoxaban) possiedono tutte le potenzialità per essere utilizzati cronicamente nella profilassi degli eventi tromboembolici ma, sfortunatamente, non esistono ancora dati pubblicati nei pazienti affetti da malattia renale cronica terminale $(56,57)$.

Solo di recente sono state valutate le caratteristiche farmacocinetiche e farmacodinamiche del dabigatran nei pazienti sottoposti al trattamento emodialitico, ma i risultati non sono ancora stati pubblicati $(58,59)$.

Gli inibitori diretti del fattore Xa sono stati studiati in pazienti con filtrato glomerulare (eGFR) compreso tra 30 e $50 \mathrm{~mL} /$ $\min / 1.73 \mathrm{~m}^{2}$ e le evidenze hanno dimostrato una minore incidenza di sanguinamento, sempre nei pazienti affetti da malattia renale cronica allo stadio pre-terminale, rispetto al dabiga$\operatorname{tran}(32,57)$.

Allo stato attuale la FDA (Food and Drug Administration) ha approvato l'impiego del dabigatran, per la profilassi tromboembolica in corso di fibrillazione atriale, alla dose di $300 \mathrm{mg} /$ die (in doppia somministrazione giornaliera da $150 \mathrm{mg}$ ognuna) nei pazienti con eGFR $>30 \mathrm{~mL} / \mathrm{min}$.

L'apixaban è tuttora in attesa dell'approvazione da parte della FDA; al momento, studi di fase 3 con un dosaggio di $5 \mathrm{mg} /$ die (in due somministrazioni giornaliere di $2.5 \mathrm{mg}$ ) in pazienti affetti da fibrillazione atriale e malattia renale cronica agli stadi 3-4a hanno evidenziato un buon profilo di sicurezza e di efficacia (57).

\section{Riassunto}

La fibrillazione atriale (FA) rappresenta la più comune forma di aritmia riscontrabile nella pratica clinica quotidiana, soprattutto nei pazienti affetti da cardiopatia ischemica cronica e da malattia renale cronica (CKD), ed è spesso correlata all'età avanzata e alla presenza di altre comorbidità, come ipertensione arteriosa, diabete mellito e vasculopatia polidistrettuale.

Negli ultimi 20 anni si è assistito a un progressivo incremento dell'incidenza della fibrillazione atriale nei pazienti affetti da malattia renale cronica, soprattutto nei pazienti sottoposti al trattamento sostitutivo della funzione renale.

Parole chiave: Malattia renale cronica, Dialisi, Cardiomiopatia dilatativa, Fibrillazione atriale

Dichiarazione di conflitto di interessi: Gli Autori dichiarano di non avere conflitto di interesse.

Contributi economici agli Autori: Gli Autori dichiarano di non aver ricevuto sponsorizzazioni economiche per la preparazione dell'articolo.

Indirizzo degli Autori:

Dr. Luca Di Lullo

U.O.C. Nefrologia e Dialisi

Ospedale L. Parodi-Delfino

Piazza A. Moro 1

00034 Colleferro (RM)

dilulloluca69@gmail.com 


\section{Bibliografia}

1. Gupta S, Figueredo VM. Tachycardia mediated cardiomyopathy: Pathophysiology, mechanisms, clinical features and management. Int J Cardiol 2014.

2. Kannel WB, Abbott RD, Savage DD, McNamara PM. Epidemiologic features of chronic atrial fibrillation: the Framingham study. N Engl J Med 1982; 306 (17): 1018-22.

3. Wolf PA, Benjamin EJ, Belanger AJ, Kannel WB, Levy D, D'Agostino RB. Secular trends in the prevalence of atrial fibrillation: the Framingham study. Am Heart J 1996; 131: 790-5.

4. Kannel WB, Wolf PA, Benjamin EJ, Levy D. Prevalence, incidence, prognosis, and predisposing conditions for atrial fibrillation: population-based estimates. Am J Cardiol 1998; 82 (8A): 2N-9N.

5. Salam AM, Albinali HA, Al-Sulaiti EM, Al-Mulla AW, Singh R, Al Suwaidi J. Effect of Age on Treatment, Trends and Outcome of Patients Hospitalized With Atrial Fibrillation: Insights From a 20-Years Registry in a Middle-eastern Country (1991-2010). Aging Clin Exp Res 2012; 24 (6): 682-90.

6. Salam AM, Albinali HA, Al-Mulla AW, et al. Women hospitalized with atrial fibrillation: Gender differences, trends and outcome from a 20-year registry in a middle eastern country (1991-2010). Int J Cardiol 2013; 168 (2): 975-80.

7. Salam AM, Albinali HA, Al-Mulla AW, Singh R, Al Suwaidi J. Secular Trends, Treatments, and Outcomes of Middle Eastern Arab and South Asian Patients Hospitalized With Atrial Fibrillation: Insights From a 20-Year Registry in Qatar (1991-2010). Angiology 2013; 64 (7): 498-504.

8. Go AS, Chertow GM, Fan D, McCulloch CE, Hsu CY. Chronic kidney disease and the risks of death, cardiovascular events, and hospitalisation. N Engl J Med 2004; 351: 1296-305.

9. Sarnak MJ, Levey AS, Schoolwerth AC, et al. Kidney disease as a risk factor for development of cardiovascular disease: a statement from the American Heart Association Councils on Kidney in Cardiovascular Disease, High Blood Pressure Research, Clinical Cardiology, and Epidemiology and Prevention. Hypertension 2003; 42 (5): 1050-65.

10. Salim I, Suwaidi JA, Ghadban W, Salam AM. Systematic Review of Atrial Fibrillation in Patients with Chronic Kidney Disease: Prevalence, Incidence, Morbidity and Mortality. Int J Cardiovasc Res 1:3.

11. Vazquez E, Sanchez-Perales C, Garcia-Garcia F, et al. Atrial fibrillation in incident dialysis patients. Kidney Int 2009; 76 (3): 324-30.

12. Ananthapanyasut W, Napan S, Rudolph EH, et al. Prevalence of atrial fibrillation and its predictors in nondialysis patients with chronic kidney disease. Clin J Am Soc Nephrol 2010; 5 (2): 173-81.

13. Soliman EZ, Prineas RJ, Go AS, et al. Chronic kidney disease and prevalent atrial fibrillation: the Chronic Renal Insufficiency Cohort (CRIC).Am Heart J 2010; 159 (6): 1102-7.

14. McManus DD, Corteville DC, Shlipak MG, Whooley MA, Ix JH. Relation of kidney function and albuminuria with atrial fibrillation (from the Heart and Soul Study). Am J Cardiol 2009; 104 (11): 1551-5.

15. Deo R, Katz R, Kestenbaum B, et al. Impaired kidney function and atrial fibrillation in elderly subjects. J Card Fail 2010; 16 (1): $55-60$.
16. Baber U, Howard VJ, Halperin JL, et al. Association of chronic kidney disease with atrial fibrillation among adults in the United States: REasons for Geographic and Racial Differences in Stroke (REGARDS) Study. Circ Arrhythm Electrophysiol 2011; 4 (1): 26-32.

17. Genovesi S, Pogliani D, Faini A, et al. Prevalence of atrial fibrillation and associated factors in a population of long-term hemodialysis patients. Am J Kidney Dis 2005; 46 (5): 897-902.

18. Genovesi S, Vincenti A, Rossi E, et al. Atrial fibrillation and morbidity and mortality in a cohort of long-term hemodialysis patients. Am J Kidney Dis 2008; 51 (2): 255-62.

19. Vázquez-Ruiz de Castroviejoa E, Sánchez-Perales C, LozanoCabezas C, et al. Incidence of atrial fibrillation in hemodialysis patients. A prospective long-term follow-up study. Rev Esp Cardiol 2006; 59 (8): 779-84.

20. Abbott KC, Trespalacios FC, Taylor AJ, Agodoa LY. Atrial fibrillation in chronic dialysis patients in the United States: risk factors for hospitalization and mortality. BMC Nephrol 2003; 4: 1 .

21. Wizemann V, Tong L, Satayathum S, et al. Atrial fibrillation in hemodialysis patients: clinical features and associations with anticoagulant therapy. Kidney Int 2010; 77 (12): 1098-106.

22. Schotten U, Verheule S, Kirchhof P, Goette A. Pathophysiological mechanisms of atrial fibrillation: a translational appraisal. Physiol Rev 2011; 91 (1): 265-325.

23. Haïssaguerre M, Jaïs P, Shah DC, et al. Spontaneous initiation of atrial fibrillation by ectopic beats originating in the pulmonary veins. N Engl J Med 1998; 339 (10): 659-66.

24. Verma A, Mantovan R, Macle L, et al. Substrate and Trigger Ablation for Reduction of Atrial Fibrillation (STAR AF): a randomized, multicentre, international trial. Eur Heart J 2010; 31 (11): 1344-56.

25. Aviles RJ, Martin DO, Apperson-Hansen C, et al. Inflammation as a risk factor for atrial fibrillation. Circulation 2003; 108 (24): 3006-10.

26. Gedikli O, Dogan A, Altuntas I, et al. Inflammatory markers according to types of atrial fibrillation. Int J Cardiol 2007; 120 (2): 193-7.

27. Koyama T, Tada H, Sekiguchi Y, et al. Prevention of atrial fibrillation recurrence with corticosteroids after radiofrequency catheter ablation: a randomized controlled trial. J Am Coll Cardiol 2010; 56 (18): 1463-72.

28. Friedrichs K, Klinke A, Baldus S. Inflammatory pathways underlying atrial fibrillation. Trends Mol Med 2011; 17 (10): 556-63.

29. Oberg BP, McMenamin E, Lucas FL, et al. Increased prevalence of oxidant stress and inflammation in patients with moderate to severe chronic kidney disease. Kidney Int 2004; 65 (3): 1009-16.

30. Cottone S, Lorito MC, Riccobene R, et al. Oxidative stress, inflammation and cardiovascular disease in chronic renal failure. J Nephrol 2008; 21 (2): 175-9.

31. Fukunaga N, Takahashi N, Hagiwara S, et al. Establishment of a model of atrial fibrillation associated with chronic kidney disease in rats and the role of oxidative stress. Heart Rhythm 2012; 9 (12): 2023-31.

32. Salim I, Al Suwaidi J, Ghadban W, Salam AM. Anticoagulation in atrial fibrillation and co-existent chronic kidney disease: efficacy versus safety. Expert Opin Drug Saf 2013; 12 (1): 53-63.

33. Wolf PA, Dawber TR, Thomas HE Jr, Kannel WB. Epidemiologic assessment of chronic atrial fibrillation and risk of stroke: the Framingham study. Neurology 1978; 28 (10): 973-7. 
34. Seliger SL, Gillen DL, Longstreth WT Jr, Kestenbaum B, Stehman-Breen CO. Elevated risk of stroke among patients with end-stage renal disease. Kidney Int 2003; 64 (2): 603-9.

35. Vázquez E, Sánchez-Perales C, Lozano C, et al. Comparison of prognostic value of atrial fibrillation versus sinus rhythm in patients on long-term hemodialysis. Am J Cardiol 2003; 92 (7): 868-71.

36. Olesen JB, Lip GY, Kamper AL, et al. Stroke and bleeding in atrial fibrillation with chronic kidney disease. N Engl J Med 2012; 367 (7): 625-35.

37. Gage BF, Waterman AD, Shannon W, Boechler M, Rich MW, Radford MJ. Validation of clinical classification schemes for predicting stroke: results from the National Registry of Atrial Fibrillation. JAMA 2001; 285 (22): 2864-70.

38. Lip GY, Nieuwlaat R, Pisters R, Lane DA, Crijns HJ. Refining clinical risk stratification for predicting stroke and thromboembolism in atrial fibrillation using a novel risk factor-based approach: the euro heart survey on atrial fibrillation. Chest 2010; 137 (2): 263-72.

39. Piccini JP, Stevens SR, Chang Y, et al. Renal dysfunction as a predictor of stroke and systemic embolism in patients with nonvalvular atrial fibrillation: validation of the R(2)CHADS(2) index in the ROCKET AF (Rivaroxaban Once-daily, oral, direct factor Xa inhibition Compared with vitamin $\mathrm{K}$ antagonism for prevention of stroke and Embolism Trial in Atrial Fibrillation) and ATRIA (AnTicoagulation and Risk factors In Atrial fibrillation) study cohorts. Circulation 2013; 127 (2): 224-32.

40. Benjamin EJ, Wolf PA, D'Agostino RB, Silbershatz H, Kannel WB, Levy D. Impact of atrial fibrillation on the risk of death: the Framingham Heart Study. Circulation 1998; 98 (10): 946-52.

41. Wiesholzer M, Harm F, Tomasec G, Barbieri G, Putz D, Balcke $\mathrm{P}$. Incidence of stroke among chronic hemodialysis patients with nonrheumatic atrial fibrillation. Am J Nephrol 2001; 21 (1): 35-9.

42. Chinitz JS, Halperin JL, Reddy VY, Fuster V. Rate or rhythm control for atrial fibrillation: update and controversies. Am J Med 2012; 125 (11): 1049-56.

43. Salam AM. Dronedarone in atrial fibrillation: the aftermath of the PALLAS trial. Expert Rev Cardiovasc Ther 2012; 10 (11): 1345-9.

44. Letavernier E, Couzi L, Delmas Y, Moreau K, Murcott O, de Précigout V. Verapamil and mild hyperkalemia in hemodialysis patients: a potentially hazardous association. Hemodial Int 2006; 10 (2): 170-2.

45. Aronow WS. Acute and chronic management of atrial fibrillation in patients with late-stage CKD. Am J Kidney Dis 2009; 53 (4): 701-10.

46. Di Loreto P, Ronco C, Vescovo G. Long QT, alteration of calcium-phosphate product, prevalence of ventricular arrhythmias and sudden death in peritoneal dialysis patients: a Holter study. Italian Journal of Medicine 2012; 6 (2): 99-104.

47. Moro C, Romero J, Corres Peiretti MA. Amiodarone and hypokalemia. A dangerous combination. Int J Cardiol 1986; 13 (3): 365-8.

48. Wazni OM, Marrouche NF, Martin DO, et al. Radiofrequency ablation vs antiarrhythmic drugs as first-line treatment of symptomatic atrial fibrillation: a randomized trial. JAMA 2005; 293 (21): 2634-40.

49. Pappone C, Augello G, Sala S, et al. A randomized trial of circumferential pulmonary vein ablation versus antiarrhythmic drug therapy in paroxysmal atrial fibrillation: the APAF Study. J Am Coll Cardiol 2006; 48 (11): 2340-7.

50. Hunter RJ, McCready J, Diab I, et al. Maintenance of sinus rhythm with an ablation strategy in patients with atrial fibrillation is associated with a lower risk of stroke and death. Heart 2012; 98 (1): 48-53.

51. Naruse Y, Tada H, Sekiguchi Y, et al. Concomitant chronic kidney disease increases the recurrence of atrial fibrillation after catheter ablation of atrial fibrillation: a mid-term follow-up. Heart Rhythm 2011; 8 (3): 335-41.

52. Hart GR, Pearce AL, Aguilar IM. Meta-analysis: antithrombotic therapy to prevent stroke in patients who have nonvalvular atrial fibrillation. Ann Intern Med 2007; 146: 857-67.

53. Knoll F, Sturm G, Lamina C, et al. Coumarins and survival in incident dialysis patients. Nephrol Dial Transplant 2012; 27 (1): 332-7.

54. Lai HM, Aronow WS, Kalen P, et al. Incidence of thromboembolic stroke and of major bleeding in patients with atrial fibrillation and chronic kidney disease treated with and without warfarin. Int J Nephrol Renovasc Dis 2009; 2: 33-7.

55. Elliott MJ, Zimmerman D, Holden RM.Warfarin anticoagulation in hemodialysis patients: a systematic review of bleeding rates. Am J Kidney Dis 2007; 50 (3): 433-40.

56. Connolly SJ, Ezekowitz MD, Yusuf S, et al. Dabigatran versus warfarin in patients with atrial fibrillation. N Engl J Med 2009; 361 (12): 1139-51.

57. Granger CB, Alexander JH, McMurray JJ, et al. Apixaban versus warfarin in patients with atrial fibrillation. N Engl J Med 2011; 365 (11): 981-92.

58. ClinicalTrials.gov (2012). Pharmacokinetics of Dabigatran Etexilate (Pradaxa ${ }^{\circledR}$ ) During Haemodialysis. Retrieved from http:// clinicaltrials.gov/ct2/show/NCT01241539?term=dabigatran\&r ank $=4$.

59. ClinicalTrials.gov (2012). An Evaluation of the Pharmacokinetics and Pharmacodynamics of Oral Dabigatran Etexilate in Hemodialysis Patients. Retrieved from http://clinicaltrials.gov/ ct2/show/NCT01590823?term=dabigatran\&rank $=5$. 